\title{
Effects of infliximab on markers of inflammation and bone turnover and associations with bone mineral density in patients with ankylosing spondylitis
}

\author{
S Visvanathan, ${ }^{1}$ D van der Heijde, ${ }^{2}$ A Deodhar, ${ }^{3}$ C Wagner, ${ }^{1}$ D G Baker, ${ }^{1}$ J Han, \\ J Braun ${ }^{4}$
}

- Additional supplemental tables 1 and 2 are published online only at http://ard.bmj. com/content/vol68/issue2

${ }^{1}$ Centocor Research and Development, Inc., Malvern, Pennsylvania, USA; ${ }^{2}$ University Hospital Maastricht, Maastricht, The Netherlands; ${ }^{3}$ Oregon Health and Science University, Portland, Oregon, USA:

${ }^{4}$ Rheumazentrum Ruhrgebiet, Herne, Germany

Correspondence to:

Sudha Visvanathan, Centocor

Research and Development, Inc., 200 Great Valley Parkway,

Mailstop RA-1-4; Malvern, PA

19355, USA:

svisvana@cntus.jni.com

Accepted 16 March 2008

Published Online First

21 May 2008

\section{ABSTRACT}

Objectives: To evaluate the relationship between bone mineral density (BMD) and biomarkers of bone turnover and inflammation in patients with ankylosing spondylitis (AS) treated with infliximab.

Methods: Patients $(n=279)$ were randomly assigned (3:8) to receive placebo or $5 \mathrm{mg} / \mathrm{kg}$ infliximab every 6 weeks through week 96. At week 24, placebo-treated patients crossed over to infliximab $5 \mathrm{mg} / \mathrm{kg}$. Starting at week 36 , patients treated with infliximab received dose escalations to $7.5 \mathrm{mg} / \mathrm{kg}$. Hip and spine BMD were measured (baseline, week 24, week 102) using dualenergy $x$-ray absorptiometry. Sera were analysed (baseline, week 24, week 102) for levels of bone alkaline phosphatase (BAP), osteocalcin, C-terminal cross-linking telopeptide of type I collagen (CTX), interleukin-6 (IL-6), vascular endothelial growth factor (VEGF) and transforming growth factor- $\beta$.

Results: Patients treated with infliximab showed significantly greater median increases in $\mathrm{BMD}$ of the spine $(2.5 \%, p<0.001)$ and hip $(0.5 \%, p=0.033)$ at week 24 than those who received placebo $10.5 \%$ and $0.2 \%$ respectively). Baseline levels of IL-6, VEGF, osteocalcin, BAP and CTX were significantly correlated with increases in spinal BMD at weeks 24 and 102 in the infliximab group. In a multiple regression analysis, high baseline osteocalcin levels and early increases in BAP at week 2 were significantly associated with increases in $\mathrm{BMD}$ scores of the spine (week 102) and hip (weeks 24 and 102) in the infliximab group

Conclusions: Patients with AS who received infliximab showed significant increases in BMD scores over 2 years. While many significant correlations were observed between BMD scores of the hip and spine and biomarker levels, high baseline osteocalcin levels and early increases in BAP were consistently associated with increases in BMD scores.

Bone formation and bone resorption, such as syndesmophytes, ankylosis and erosions, are features of progressive ankylosing spondylitis (AS). However, studies of biochemical markers of bone turnover in patients with AS have yielded conflicting results. Some studies show normal ${ }^{12}$ or low $^{34}$ levels of bone formation markers, such as osteocalcin or bone alkaline phosphatase (BAP), while other studies show elevated levels of these biomarkers. ${ }^{5}$ Studies examining markers of bone resorption (eg, pyridinium cross-linking telopeptide of type I collagen) in patients with AS also yield conflicting results. ${ }^{16-9}$ However, elevated levels of bone resorption markers have been shown to correlate with inflammatory markers and higher levels of acute-phase reactants in patients with $\mathrm{AS}^{1}$ as well as with inflammatory markers in patients with osteoporosis. ${ }^{10}$

In this study, we evaluated changes in the levels of markers associated with bone turnover and inflammation in patients with AS who received infliximab in the Ankylosing Spondylitis Study for the Evaluation of Recombinant Infliximab Therapy (ASSERT). We also determined the relationship between baseline levels and changes in these markers and increases in bone mineral density (BMD) after initiation of infliximab therapy. We hypothesise that changes in these biochemical markers after treatment with infliximab would be associated with an improvement in the disease processes corresponding with reduced inflammation and increased bone growth.

\section{METHODS}

Details of the ASSERT study have been previously published. ${ }^{11}{ }^{12}$ Briefly, patients with AS for at least 3 months before screening were randomly assigned $(3: 8)$ to infusions of placebo or $5 \mathrm{mg} / \mathrm{kg}$ infliximab at weeks $0,2,6,12$ and 18. At week 24, patients assigned to placebo crossed over to $5 \mathrm{mg} / \mathrm{kg}$ infliximab, and both groups continued treatment every 6 weeks through week 96 . Patients originally assigned to infliximab could receive dose escalations up to $7.5 \mathrm{mg} / \mathrm{kg}$ starting at week 36 if their Bath Ankylosing Spondylitis Disease Activity Index score was at least 3 for two consecutive visits.

Sera from patients were collected for biomarker testing at weeks 0, 2, 24 and 102. BAP (Quidel, San Diego, California, USA), osteocalcin (Nordic Biosciences, Herlev, Denmark), and C-terminal cross-linking telopeptide of type I collagen (CTX) (Nordic Biosciences) were evaluated as markers of bone turnover. Interleukin-6 (IL-6), vascular endothelial growth factor (VEGF), and transforming growth factor- $\beta$ (TGF- $\beta$ ) were evaluated as markers of inflammation (R\&D Systems, Minneapolis, USA).

The BMD of patients aged 20-84 $(\mathrm{n}=276)$ was measured by dual-energy $x$-ray absorptiometry (DEXA) evaluations of the hip and spine (L1-L4) at baseline, week 24 and week 102. Vertebrae exhibiting abnormalities (eg, fracture or surgical alteration) were excluded from BMD analysis. Osteopenia was defined as a $\mathrm{T}$ score between -2.5 and -1 , exclusive. Osteoporosis was defined as a $\mathrm{T}$ score of -2.5 or lower. 
The presence of syndesmophytes was determined using radiography at baseline and week $102 .{ }^{13}$ Briefly, lateral radiographs of the cervical and lumbar spine were scored using the modified Stoke Ankylosing Spondylitis Spine Score (mSASSS). ${ }^{14} 15$ The anterior sites of the lower and upper portion of each vertebra were scored from the T12 lower border to the S1 upper border for the lumbar spine and from the C2 lower border to the T1 upper border for the cervical spine. Each of the 24 regions was assigned a score of 0 (normal), 1 (erosion, sclerosis, or squaring), 2 (syndesmophyte) or 3 (bridging syndesmophyte). Syndesmophytes were defined as regional scores of 2 or greater. A patient who had a vertebral region score less than 2 at baseline and greater than or equal to 2 at week 102 was considered to have developed a syndesmophyte.

\section{Statistical analyses}

This study is post hoc and exploratory; therefore, the statistics are descriptive only and are not adjusted for multiplicity. Changes from baseline to weeks 2 and 24 in biomarker levels and changes from baseline to week 24 in BMD scores, $T$ scores and $Z$ scores of the hip and spine were evaluated using an analysis of variance on the van der Waerden normal scores. Univariate correlations between individual biomarkers (at baseline and week 24) and between biomarker levels (at baseline and per cent change from baseline to weeks 2 and 24) and changes from baseline to weeks 24 and 102 in spinal and hip BMD scores were determined using Spearman's ranking correlation coefficient. Multiple linear regression analyses were performed using baseline and change from baseline to week 2 in biomarker levels (IL-6, VEGF, BAP, osteocalcin and TGF- $\beta$ ), syndesmophyte development (from baseline to week 102), and change from baseline to weeks 24 and 102 in BMD scores (hip and spine) to determine whether serum biomarker levels or syndesmophyte development were significantly associated with increases in BMD scores of the spine or hip. Box plots were used to depict the actual and per cent changes from baseline in $\mathrm{BMD}$ and actual change from baseline in T scores at weeks 24 and 102. Statistical analyses were performed using the SAS system (SAS Institute, Cary, North Carolina, USA).

\section{RESULTS}

Baseline patient characteristics were comparable between treatment groups (table 1).

\section{Correlations between biomarker levels}

Spearman correlation coefficients ( $r$ ) among biomarker levels at baseline and week 24 are summarised in table 2 .

At baseline, the strongest relationship was observed between the markers of bone turnover osteocalcin and CTX ( $r=0.476$, $\mathrm{p}<0.0001)$. Significant but modest correlations were observed between the markers of bone formation BAP and osteocalcin $(r=0.336, p<0.0001)$ and between BAP and CTX $(r=0.367$, $\mathrm{p}<0.0001)$. Significant but modest correlations among inflammatory markers were observed between IL-6 and VEGF $(r=0.264, \quad p<0.0001)$ and between VEGF and TGF- $\beta$ $(\mathrm{r}=0.256, \mathrm{p}<0.0001)$.

At week 24 in the infliximab group, the strongest correlations were observed between IL- 6 and VEGF $(r=0.446, p<0.0001)$ and between VEGF and TGF- $\beta(r=0.494, p<0.0001)$. Modest correlations were observed between BAP and osteocalcin $(\mathrm{r}=0.391, \mathrm{p}<0.0001), \quad \mathrm{IL}-6$ and osteocalcin $(\mathrm{r}=-0.268$, $\mathrm{p}<0.005)$, VEGF and osteocalcin $(\mathrm{r}=-0.288, \mathrm{p}<0.005)$, and CTX and osteocalcin $(r=0.273, p<0.005)$. In the placebo group at week 24, significant correlations were observed between TGF- $\beta$ and VEGF $(r=0.564, p<0.0001)$, and modest correlations were observed between osteocalcin and CTX $(r=0.259$, $\mathrm{p}<0.05)$ and between osteocalcin and BAP $(r=0.274, p<0.05)$.

\section{Changes in biomarker levels following infliximab treatment}

Percentage changes in biomarker levels from baseline to weeks 2, 24 and 102 are shown in fig 1. A significantly greater median per cent increase in BAP was observed in the infliximab group at week $2(5.8 \%)$ compared with the placebo group $(-0.6 \%$, $\mathrm{p}=0.013)$. The median per cent increase in BAP from baseline to week 24 in the infliximab group was similar to the value at week $2(6.0 \%)$, but greater variability was observed in both the infliximab and placebo groups, and the difference between groups was not significant $(p=0.085)$. Trends toward a greater increase in osteocalcin at week $24(\mathrm{p}=0.063)$ and a greater reduction in TGF- $\beta$ at weeks $2(p=0.059)$ and $24(p=0.091)$ were observed among patients treated with infliximab. No differences were observed between the treatment groups in the changes in CTX levels from baseline to weeks 2 or 24.

After 2 years of therapy, greater median reductions in serum levels of TGF- $\beta$ and CTX and greater median increases in BAP levels were observed as compared with weeks 2 and 24 (fig 1). Changes in serum marker levels for patients treated with placebo who crossed over to infliximab at week 24 were similar to those for patients who were originally assigned to receive infliximab.

The significant reductions from baseline to weeks 2 and 24 in serum levels of IL- 6 and VEGF observed in patients treated with infliximab compared with patients treated with placebo in the ASSERT study have been previously reported. ${ }^{16}$

\section{Changes in bone mineral density following infliximab treatment} At week 24, significant increases from baseline in BMD scores were observed in the spine $(p<0.001)$ and hip $(p=0.040)$ of patients in the infliximab group (fig $2 \mathrm{~A}$ ). The corresponding median per cent increase in spinal BMD scores was $2.5 \%$ in patients treated with infliximab and $0.5 \%$ in patients treated with placebo $(p<0.001)$, and the median per cent increase in hip $\mathrm{BMD}$ scores was $0.5 \%$ in patients treated with infliximab and $0.2 \%$ in patients treated with placebo $(p=0.033)$ (fig $2 B$ ). Similar increases were observed in the $T$ scores of the spine $(p<0.001)$ and hip $(p=0.017)$ at week 24 (fig 2C). Changes from baseline to week 102 in spine and hip $\mathrm{T}$ scores and total $\mathrm{BMD}$ increased further over the week 24 values for the infliximab group. The median per cent increase in spinal BMD scores was $6.8 \%$ in the infliximab group and $4.1 \%$ in the placebo/infliximab group; the median per cent increase in hip $\mathrm{BMD}$ scores was $1.8 \%$ and $0.9 \%$ respectively (fig $2 \mathrm{~B}$ ). Supplemental table 1 summarises the proportions of patients with osteopenia or osteoporosis at baseline and weeks 24 and 102.

\section{Syndesmophytes}

At baseline, approximately two-thirds of all patients had syndesmophytes as detected by the analysis of radiographs (table 1). No statistically significant differences were observed in the percentage increase in BMD from baseline to week 102 for patients who developed 1 or more syndesmophytes compared with those who did not, from baseline to week 102 (supplemental table 2). 
Table 1 Baseline characteristics

\begin{tabular}{|c|c|c|}
\hline & Placebo $(n=78)$ & $5 \mathrm{mg} / \mathrm{kg}$ infliximab $(\mathrm{n}=201)$ \\
\hline Men, no. (\%) & $68(87.2 \%)$ & $157(78.1 \%)$ \\
\hline \multicolumn{3}{|l|}{ Age, years } \\
\hline Mean (SD) & $40.3(9.4)$ & $39.6(10.6)$ \\
\hline \multicolumn{3}{|l|}{ Disease duration, years } \\
\hline $\mathrm{N}$ & 76 & 201 \\
\hline Mean (SD) & $11.9(8.0)$ & $10.1(8.7)$ \\
\hline Median (range) & $13.2(0.3,31.6)$ & $7.7(0.3,41.1)$ \\
\hline \multicolumn{3}{|l|}{ HLA-B27 positive } \\
\hline $\mathrm{N}$ & 78 & 200 \\
\hline №. (\%) & $69(88.5 \%)$ & $173(86.5 \%)$ \\
\hline \multicolumn{3}{|c|}{$\begin{array}{l}\text { Inflammation (average morning stiffness on a visual } \\
\text { analogue scale } 0-10 \mathrm{~cm} \text { ) }\end{array}$} \\
\hline $\mathrm{N}$ & 78 & 201 \\
\hline Mean (SD) & $6.9(1.9)$ & $6.9(2.3)$ \\
\hline Median (range) & $7.0(0.8,10.0)$ & $7.3(0.2,10.0)$ \\
\hline \multicolumn{3}{|l|}{ Bone mineral density } \\
\hline \multicolumn{3}{|c|}{ Total spine bone mineral density $\left(\mathrm{g} / \mathrm{cm}^{2}\right)$} \\
\hline $\mathrm{N}$ & 73 & 189 \\
\hline Mean (SD) & $1.09(0.25)$ & $1.01(0.18)$ \\
\hline Median (range) & $1.02(0.77,2.01)$ & $1.01(0.63,1.64)$ \\
\hline \multicolumn{3}{|l|}{ Spine T scores } \\
\hline $\mathrm{N}$ & 72 & 186 \\
\hline Mean (SD) & $-0.28(2.00)$ & $-0.96(1.45)$ \\
\hline Median (range) & $-0.75(-2.70,7.40)$ & $-0.95(-4.20,3.60)$ \\
\hline \multicolumn{3}{|c|}{ Total hip bone mineral density $\left(\mathrm{g} / \mathrm{cm}^{2}\right)$} \\
\hline $\mathrm{N}$ & 74 & 192 \\
\hline Mean (SD) & $0.95(0.14)$ & $0.92(0.14)$ \\
\hline Median (range) & $0.92(0.69,1.36)$ & $0.91(0.59,1.38)$ \\
\hline \multicolumn{3}{|l|}{ Hip T scores } \\
\hline $\mathrm{N}$ & 74 & 190 \\
\hline Mean (SD) & $-0.62(0.91)$ & $-0.75(0.97)$ \\
\hline Median (range) & $-0.70(-2.20,2.10)$ & $-0.80(-3.30,2.30)$ \\
\hline \multicolumn{3}{|l|}{ Hip Z scores } \\
\hline $\mathrm{N}$ & 74 & 190 \\
\hline Mean (SD) & $-0.42(0.90)$ & $-0.54(0.95)$ \\
\hline Median (range) & $-0.60(-2.00,2.24)$ & $-0.60(-3.00,2.50)$ \\
\hline \multicolumn{3}{|l|}{ Syndesmophytes } \\
\hline Patients with syndesmophytes* & $54(71.1 \%)$ & $122(61.0 \%)$ \\
\hline Regions with syndesmophytes $\dagger$ & $486(27.6 \%)$ & 908 (19.3\%) \\
\hline \multicolumn{3}{|l|}{ Biomarker levels } \\
\hline \multicolumn{3}{|l|}{ IL-6 (pg/ml) } \\
\hline $\mathrm{N}$ & 67 & 187 \\
\hline No. (\%) with values $\geqslant$ LLO0 & $32(47.8 \%)$ & $105(56.1 \%)$ \\
\hline Mean (SD) & $11.4(17.2)$ & $13.2(20.0)$ \\
\hline Median (range) & $3.1(3.1,119.1)$ & $7.7(3.1,174.6)$ \\
\hline \multicolumn{3}{|l|}{ VEGF (pg/ml) } \\
\hline $\mathrm{N}$ & 75 & 193 \\
\hline No. $(\%)$ with values $\geqslant L L O 0$ & $75(100 \%)$ & $191(99.0 \%)$ \\
\hline Mean (SD) & $556.2(385.6)$ & $520.4(361.5)$ \\
\hline Median (range) & $473.5(67.8,2353.7)$ & $421.5(29.8,2067.5)$ \\
\hline \multicolumn{3}{|l|}{ TGF- $\beta$ (pg/ml) } \\
\hline $\mathrm{N}$ & 75 & 192 \\
\hline No. (\%) with values $\geqslant L L O 0$ & $75(100 \%)$ & $192(100 \%)$ \\
\hline Mean (SD) & $45.9(11.5)$ & $46.8(11.7)$ \\
\hline Median (range) & $46.7(9.4,85.9)$ & $48.1(6.0,90.3)$ \\
\hline \multicolumn{3}{|l|}{ Osteocalcin (ng/ml) } \\
\hline $\mathrm{N}$ & 73 & 197 \\
\hline No. (\%) with values $\geqslant L L O 0$ & $71(97.3 \%)$ & $197(100 \%)$ \\
\hline Mean (SD) & $17.4(8.7)$ & $18.4(9.9)$ \\
\hline Median (range) & $15.8(1.7,41.3)$ & $16.9(4.8,73.5)$ \\
\hline BAP (U/I) & & \\
\hline $\mathrm{N}$ & 73 & 197 \\
\hline No. (\%) with values $\geqslant L L O 0$ & $73(100 \%)$ & $197(100 \%)$ \\
\hline
\end{tabular}


Table 1 Continued

\begin{tabular}{|c|c|c|}
\hline & Placebo $(n=78)$ & $5 \mathrm{mg} / \mathrm{kg}$ infliximab $(\mathrm{n}=201)$ \\
\hline Mean (SD) & $27.6(10.1)$ & $26.7(9.2)$ \\
\hline Median (range) & $25.4(8.5,63.5)$ & $25.0(10.9,62.1)$ \\
\hline \multicolumn{3}{|l|}{ CTX (ng/ml) } \\
\hline $\mathrm{N}$ & 72 & 198 \\
\hline No. $(\%)$ with values $\geqslant$ LLO0 & $66(90.4 \%)$ & $184(93.4 \%)$ \\
\hline Mean (SD) & $0.39(0.24)$ & $0.39(0.25)$ \\
\hline Median (range) & $0.34(0.1,1.6)$ & $0.33(0.1,1.8)$ \\
\hline
\end{tabular}

\section{Associations between biomarker levels and changes in bone mineral density of the spine}

Univariate correlations

In the infliximab group, high baseline levels of IL- $6(r=0.146$, $p=0.0489), \quad$ VEGF $(r=0.146, \quad p=0.0453), \quad$ osteocalcin $(r=0.262, p=0.0002)$, BAP $(r=0.262, p=0.0002)$ and CTX $(r=0.189, p=0.0088)$ correlated significantly with increases from baseline to week 24 in spinal BMD scores. Decreases from baseline to weeks 2 and 24 in IL-6 $(r=-0.192, p=0.0111$ and $r=-0.206, p=0.0073$ respectively) and VEGF $(r=-0.245$, $p=0.0007$ and $r=-0.226, p=0.0025$ respectively) and increases from baseline to week 24 in BAP $(r=0.153$, $\mathrm{p}=0.0382$ ) also correlated with increases in spinal BMD scores from baseline to week 24 . In the placebo group, no significant correlations were observed between baseline biomarker levels and increases in spinal BMD scores at week 24. However, decreases from baseline to week 2 in IL-6 ( $r=-0.258$, $\mathrm{p}=0.0392)$ and increases from baseline to week 24 in CTX $(\mathrm{r}=0.241, \mathrm{p}=0.0479)$ correlated with increases in spinal BMD scores at week 24.

Data for both treatment groups were combined to evaluate correlations between BMD scores and biomarker levels and changes from baseline to week 102 because all patients were receiving infliximab after placebo crossover to infliximab at week 24. Results for the combined group at week 102 were similar to week 24. High baseline levels of IL-6 $(r=0.207$, $p=0.0030)$, VEGF $(r=0.181, p=0.0077)$, TGF- $\beta(r=0.143$, $p=0.0356)$ and CTX $(r=0.179, p=0.0084)$ correlated moderately with increases in spinal BMD scores at week 102. The strongest relationships were between the increase in spinal BMD scores at week 102 and high baseline osteocalcin $(\mathrm{r}=0.335, \mathrm{p}<0.0001)$ and BAP $(\mathrm{r}=0.251, \mathrm{p}=0.0002)$ levels. Decreases in IL-6 $(r=-0.255, p=0.0003)$ and VEGF $(r=-0.330, p<0.0001)$ and increases in BAP $(r=0.206$, $p=0.0029)$ at week 2 correlated with increases in spinal BMD scores at week 102. A similar pattern was observed for changes at week 24 in the levels of IL-6 $(r=-0.285$, $\mathrm{p}<0.0001)$, VEGF $(\mathrm{r}=-0.280, \mathrm{p}<0.0001)$ and BAP $(r=0.211, p=0.0024)$. Additionally, there was a weak but statistically significant correlation between increases in osteocalcin at week 24 and increases in spinal BMD scores at week $102(r=0.168, p=0.0165)$. Similar changes were observed when data for only patients with syndesmophytes at baseline were included in the analysis (data not shown).

Table 2 Correlations $(r)$ between biomarker values at baseline for all patients and at week 24 for patients in the infliximab and placebo groups separately

\begin{tabular}{|c|c|c|c|c|c|}
\hline & VEGF & TGF- $\beta$ & CTX & Osteocalcin & BAP \\
\hline \multicolumn{6}{|l|}{ Baseline } \\
\hline IL-6 & $0.264^{* * *}$ & 0.105 & 0.112 & 0.064 & 0.040 \\
\hline VEGF & & $0.256^{* * *}$ & 0.052 & 0.014 & 0.084 \\
\hline TGF- $\beta$ & & & 0.034 & 0.089 & $0.148^{*}$ \\
\hline CTX & & & & $0.476^{* * *}$ & $0.367^{* * *}$ \\
\hline Osteocalcin & & & & & $0.336^{* * *}$ \\
\hline \multicolumn{6}{|c|}{ Week 24: infliximab } \\
\hline IL-6 & $0.446 * * *$ & 0.123 & 0.035 & $-0.268^{* *}$ & $-0.157^{*}$ \\
\hline VEGF & & $0.494^{* * *}$ & 0.021 & $-0.288^{* *}$ & $-0.148^{* * * *}$ \\
\hline TGF- $\beta$ & & & 0.074 & 0.051 & 0.095 \\
\hline CTX & & & & $0.273^{* *}$ & $0.169^{*}$ \\
\hline Osteocalcin & & & & & $0.391^{* * *}$ \\
\hline \multicolumn{6}{|c|}{ Week 24: placebo } \\
\hline IL-6 & -0.019 & -0.041 & 0.031 & -0.130 & -0.114 \\
\hline VEGF & & $0.564^{* * *}$ & 0.102 & -0.058 & 0.087 \\
\hline TGF- $\beta$ & & & 0.162 & -0.121 & 0.071 \\
\hline CTX & & & & $0.259^{*}$ & -0.062 \\
\hline Osteocalcin & & & & & $0.274^{*}$ \\
\hline
\end{tabular}

BAP, bone alkaline phosphatase; CTX, C-terminal cross-linking telopeptide of type I collagen; IL-6, interleukin-6; TGF- $\beta$, transforming growth factor $\beta$; VEGF, vascular endothelial growth factor.

${ }^{*} \mathrm{p}<0.05$; ${ }^{* *} \mathrm{p}<0.005 ;{ }^{* * *} \mathrm{p}<0.0001 ;{ }^{* * * *} \mathrm{p}=0.0513$ 
Figure 1 Percentage changes in biomarker levels at weeks 2, 24 and 102 for transforming growth factor $\beta(A)$, osteocalcin (B), bone alkaline phosphatase $(\mathrm{C})$, and C-terminal crosslinking telopeptide of type 1 collegen (D). Boxes indicate medians; error bars indicate interquartile ranges.
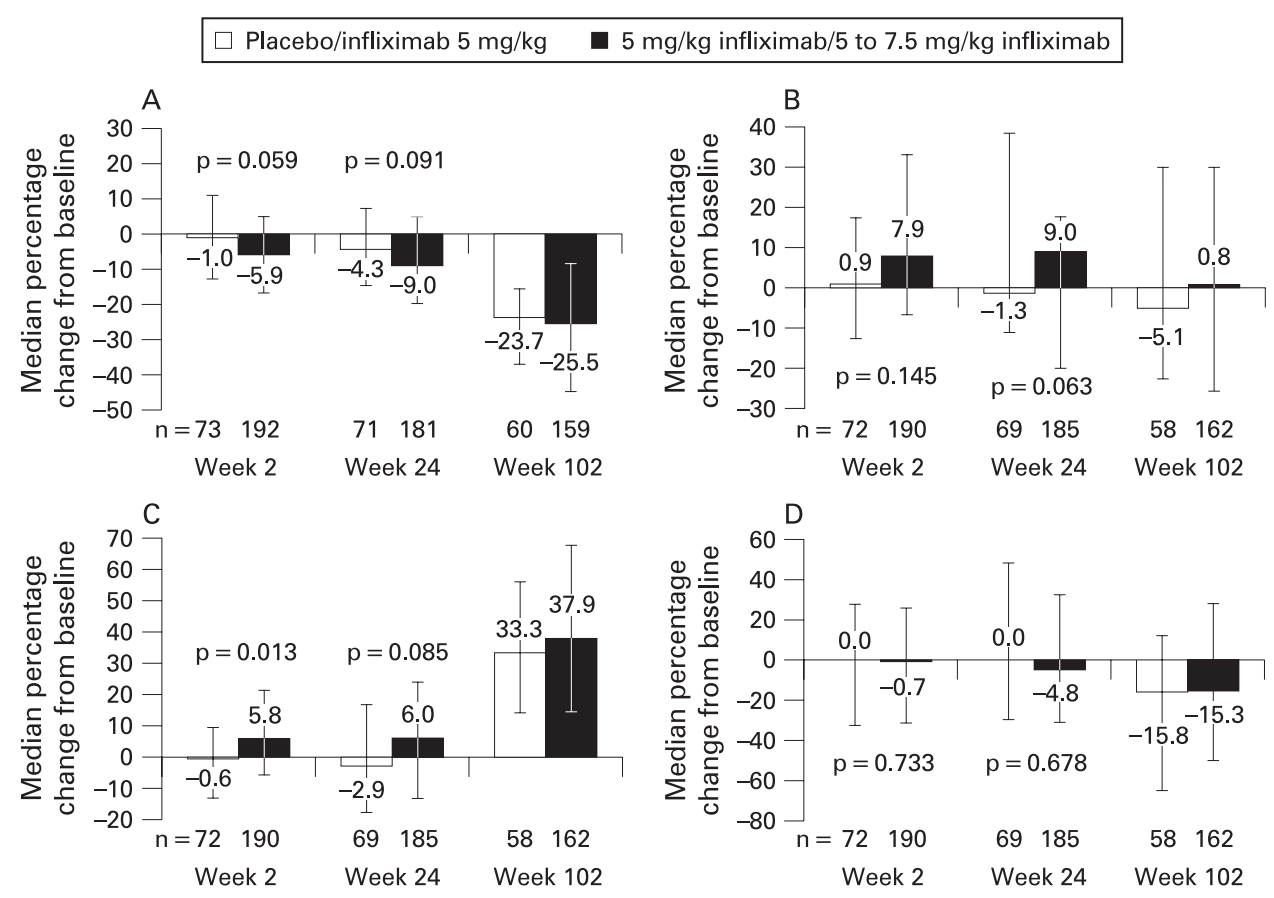

\section{Multiple regression analyses}

In the infliximab group, results of a multiple linear regression analysis using a model including baseline biomarker levels (IL-6, VEGF, TGF- $\beta$, osteocalcin, BAP and CTX), syndesmophyte development (from baseline to week 102), and changes from baseline to week 24 in spinal BMD scores revealed that none of the variables were significantly associated with increases in spinal BMD scores (table 3). Similarly, per cent changes from baseline to week 2 in these biomarkers and syndesmophyte development were not significantly associated with changes from baseline to week 24 in spinal BMD scores.

Further, in a model that included baseline biomarker levels (IL-6, VEGF, TGF- $\beta$, osteocalcin, BAP, CTX), syndesmophyte development (from baseline to week 102), and changes in spinal BMD scores from baseline to week 102, osteocalcin was the only significant variable associated with increases in spinal BMD scores. In contrast, the results of a regression analysis using a model that included changes from baseline to week 2 in biomarker levels (IL-6, VEGF, TGF- $\beta$, osteocalcin, BAP, CTX), syndesmophyte development (from baseline to week 102), and changes from baseline to week 102 in spinal BMD scores revealed that BAP, CTX and VEGF were the only significant variables associated with increases in spinal BMD scores. None of the other markers were significantly associated with increases in spinal BMD scores.

\section{Associations between biomarker levels and changes in bone mineral density of the hip}

Univariate correlations

In the univariate analysis, no baseline biomarker levels correlated with increases from baseline to week 24 in hip $\mathrm{BMD}$ scores for either treatment group. However, in the combined group, high baseline levels of TGF- $\beta \quad(r=0.170$, $p=0.0140), \quad$ CTX $\quad(r=0.173, \quad p=0.0120), \quad$ osteocalcin $(r=0.209, p=0.0024)$ and BAP $(r=0.231, p=0.0008)$ correlated significantly with increases in hip $\mathrm{BMD}$ scores from baseline to week 102 .
In the placebo group, there were no significant correlations between changes in biomarker levels from baseline to weeks 2 or 24 and increases from baseline to week 24 in hip BMD scores. In the infliximab group, decreases from baseline in VEGF at weeks 2 and $24(\mathrm{r}=-0.201, \mathrm{p}=0.0061$ and $\mathrm{r}=-0.156, \mathrm{p}=0.0379$ respectively) and increases in BAP from baseline to week 2 $(\mathrm{r}=0.187, \mathrm{p}=0.0111)$ correlated significantly with increases from baseline in hip BMD scores at week 24. Similarly, in the combined group, decreases in VEGF from baseline to weeks 2 and $24(\mathrm{r}=-0.187, \mathrm{p}=0.0071$ and $\mathrm{r}=-0.232, \mathrm{p}=0.0010$ respectively) and increases from baseline to week 2 in BAP $(r=0.154, p=0.0286)$ correlated significantly with increases from baseline to week 102 in hip BMD scores. Also in the combined group, decreases from baseline to week 24 in IL-6 $(r=-0.155, p=0.0348)$ and TGF- $\beta(r=-0.173, p=0.0153)$ and increases from baseline to week 24 in osteocalcin $(r=0.155$, $\mathrm{p}=0.0295$ ) correlated with increases in hip BMD scores from baseline to week 102. Similar changes were observed when data for only patients with syndesmophytes at baseline were included in the analysis (data not shown).

\section{Multiple regression analyses}

In the infliximab group, results of the multiple linear regression analysis using a model that included baseline biomarker levels (IL-6, VEGF, TGF- $\beta$, osteocalcin, BAP and CTX), syndesmophyte development (from baseline to week 102), and changes from baseline to week 24 in hip BMD scores revealed that osteocalcin was the only significant variable associated with increases in hip BMD scores (table 3). Osteocalcin was also the only significant variable associated with increases in hip BMD scores at week 102 using a model that included baseline biomarker levels (IL-6, VEGF, TGF- $\beta$, osteocalcin, BAP, CTX) and syndesmophyte development (from baseline to week 102). In contrast, in the infliximab group, the results of a multiple linear regression analysis using a model that included changes from baseline to week 2 in serum markers (IL-6, VEGF, TGF- $\beta$, osteocalcin, BAP, CTX), syndesmophyte development (from baseline to week 102), and changes from baseline to weeks 24 
Figure 2 Actual $(A)$ and per cent $(B)$ change from baseline in bone mineral density (BMD) and actual change from baseline $(C)$ in $T$ scores. Solid horizontal lines indicate medians, dotted horizontal lines indicate means, boxes indicate interquartile ranges, and error bars indicate standard deviations.
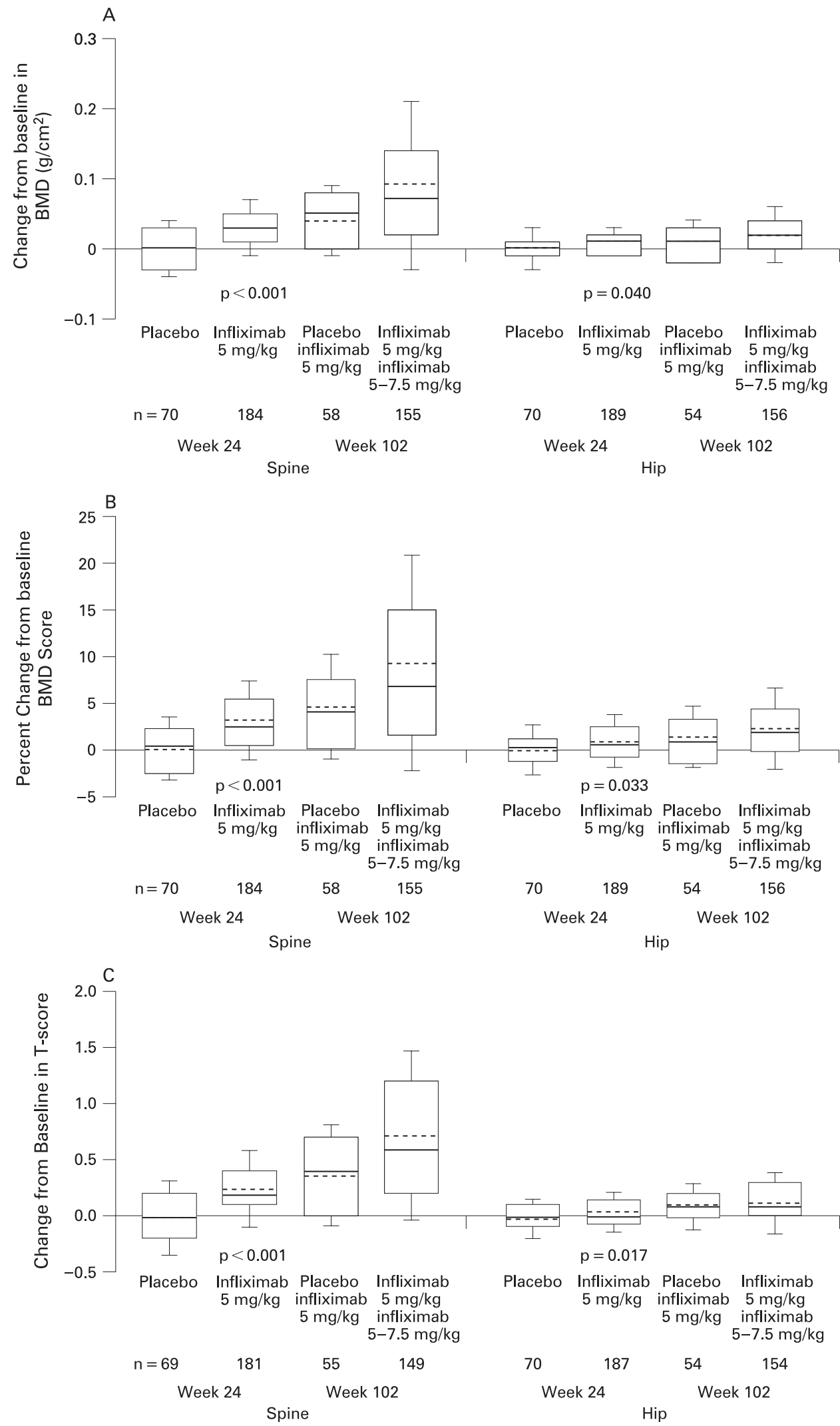

and 102 in hip BMD scores revealed that BAP and CTX were the only significant variables associated with increases in hip BMD scores at week 24 (table 3). BAP was also significantly associated with increases in hip BMD scores at week 102. None of the other markers were significantly associated with increases in hip BMD scores.

\section{DISCUSSION}

To our knowledge, these results represent the largest randomised controlled study of the effect of an antitumour necrosis factor antibody agent on BMD measurements (spine and hip). Increases in $\mathrm{BMD}$ following treatment with infliximab have been previously reported for patients with spondyloarthropathy, ${ }^{17}$ 
Table 3 Associations between biomarker levels and the change from baseline to weeks 24 and 102 in bone mineral density for patients in the infliximab group

\begin{tabular}{|c|c|c|c|}
\hline Model & $\mathbf{r}^{2}$ & $\mathbf{b}^{*}$ & p Value \\
\hline \multicolumn{4}{|l|}{ Spine BMD: \% change from baseline to week 24} \\
\hline Baseline biomarker levels, $n=144$ & NS & NS & NS \\
\hline$\%$ change from baseline to week 2 in biomarker levels, $n=135$ & NS & NS & NS \\
\hline \multicolumn{4}{|l|}{ Spine BMD: \% change from baseline to week 102} \\
\hline Baseline biomarker levels, $n=141$ & 0.111 & & \\
\hline Osteocalcin & & 0.254 & 0.019 \\
\hline$\%$ change from baseline to week 2 in biomarker levels, $n=132$ & 0.174 & & \\
\hline BAP & & 0.138 & 0.003 \\
\hline CTX & & -0.035 & 0.024 \\
\hline VEGF & & -0.081 & 0.046 \\
\hline \multicolumn{4}{|l|}{ Hip BMD: \% change from baseline to week 24} \\
\hline Baseline biomarker levels, $n=143$ & 0.153 & & \\
\hline Osteocalcin & & 0.084 & 0.002 \\
\hline$\%$ change from baseline to week 2 in biomarker levels, $n=135$ & 0.121 & & \\
\hline BAP & & 0.027 & 0.019 \\
\hline CTX & & -0.008 & 0.038 \\
\hline \multicolumn{4}{|l|}{ Hip BMD: \% change from baseline to week 102} \\
\hline Baseline biomarker levels, $n=141$ & 0.237 & & \\
\hline Osteocalcin & & 0.172 & $<0.0001$ \\
\hline$\%$ change from baseline to week 2 in biomarker levels, $n=133$ & 0.111 & & \\
\hline BAP & & 0.038 & 0.039 \\
\hline
\end{tabular}

rheumatoid arthritis, ${ }^{18} 19$ and Crohn's disease. ${ }^{20}$ In our study, greater increases in both spine and hip $\mathrm{BMD}$ scores were observed at week 24 in patients who received infliximab compared with placebo. The differences between the groups were greater for the spine than for the hip. Placebo-treated patients who crossed over to infliximab at week 24 showed increases in $\mathrm{BMD}$ scores at week 102; however, the magnitude of increase in $\mathrm{BMD}$ for patients initially assigned to infliximab treatment was greater at week 102 , suggesting a benefit from earlier treatment with infliximab.

In the current study, treatment with infliximab resulted in stronger correlations between IL- 6 and VEGF and between VEGF and TGF- $\beta$ but weaker correlations between CTX and osteocalcin and between CTX and BAP. These results suggest that changes in levels of IL-6, VEGF and TGF- $\beta$ are associated with the changes in disease processes that occur with infliximab treatment in AS. Significant univariate correlations were observed between baseline osteocalcin and both increases in spinal BMD scores at weeks 24 and 102 and increases in hip BMD scores at week 102 in the infliximab group. Further, in the multiple regression analysis that included all biomarkers and syndesmophyte development, only baseline osteocalcin levels were consistently associated with increases in spinal and hip BMD scores at week 102 for patients in the infliximab group. Baseline osteocalcin levels were also associated with increases in $\mathrm{BMD}$ hip scores at week 24. In comparison, two small-scale studies lacking follow-up of treatment effects in patients with AS did not show a relationship between osteocalcin levels and BMD scores. ${ }^{21} 22$ Our results suggest that baseline osteocalcin levels may have utility for predicting increases in hip and spinal $\mathrm{BMD}$ scores among patients with AS receiving infliximab.

Infliximab treatment significantly increased the levels of the bone formation marker BAP; although, the magnitude of median increases from baseline at weeks 2 and 24 were small (approximately 6\%), with wide variability among patients within each treatment group. Greater increases from baseline in BAP were observed at week 102 (33-38\%), but these changes cannot be specifically attributed to infliximab because the study was not placebo-controlled after week 24. Further, significant univariate correlations were observed in the infliximab group between baseline BAP and both increases in spinal BMD scores at weeks 24 and 102 and increases in hip BMD scores at week 102. The results of a multiple regression analysis showed that early increases from baseline to week 2 in BAP were significantly associated with increases in BMD hip scores at week 24 and increases in both spinal and hip BMD scores at week 102 in the infliximab group.

Treatment with infliximab in patients with Crohn's disease has been previously shown to result in a significant increase in BAP but not in N-telopeptide of type I collagen, a marker of bone resorption. ${ }^{23}$ The increases in BAP might be a result of tumour necrosis factor- $\alpha$ inhibition and the steroid-sparing effect of infliximab in Crohn's disease. However, this explanation is not applicable to our analysis given that patients were prohibited from corticosteroid use in ASSERT. In contrast, a study of patients with rheumatoid arthritis treated with infliximab showed a significant decrease in $\mathrm{N}$ telopeptide of type I collagen levels without an associated increase in BAP levels after treatment, ${ }^{24}$ supporting a disconnect between these markers of bone formation and resorption. These results suggest that BAP may be a good marker of early changes in spine and hip $\mathrm{BMD}$ scores in response to treatment with infliximab.

We have shown previously that treatment with infliximab results in significant, early decreases in the inflammatory markers IL-6 and VEGF in patients with AS as compared with placebo. ${ }^{16}$ In our study, early median decreases in TGF- $\beta$ were greater in the infliximab group than in the placebo group, but this difference was not statistically significant. Significant univariate correlations in the infliximab group were observed between early decreases in IL- 6 and VEGF levels and increases in spinal BMD scores at weeks 24 and 102, and early decreases in VEGF were also associated with increases in hip BMD scores at weeks 24 and 102. Additionally, baseline VEGF levels correlated 
significantly with increases in spinal BMD scores from baseline to weeks 24 and 102, and baseline TGF- $\beta$ levels correlated significantly with increases in hip $\mathrm{BMD}$ scores from baseline to week 102.

Increases in $\mathrm{BMD}$ scores in patients with AS may be confounded by the formation of syndesmophytes. However, in this study, patients who developed syndesmophytes had similar changes in BMD as those who did not develop syndesmophytes. Further, syndesmophyte development was not a significant variable associated with changes in either hip or spinal BMD scores at week 24 or 102 in our multiple regression models. Thus, the increases in $\mathrm{BMD}$ scores observed in patients treated with infliximab do not appear to be linked to syndesmophyte development in this study.

There are several important limitations to this study. This secondary analysis from the ASSERT study was not powered to assess the significance of relationships between changes in biomarker levels and the BMD of patients with AS who received infliximab. Changes in BMD were only assessed at two time points (weeks 24 and 102), and changes in biomarker levels were only assessed at three time points (weeks 2, 24 and 102); fluctuations of these variables between time points were not studied. A more frequent, and perhaps longer, follow-up of patients would provide a more complete understanding of the long-term effects of infliximab therapy on both fluctuations in biomarker levels and BMD of the spine and hip in patients with AS. The variability in the changes in biomarker levels, particularly at weeks 2 and 24, limits the utility of these surrogate markers of $\mathrm{BMD}$ at the individual patient level.

In conclusion, patients with AS who received infliximab showed significant increases in BMD of the hip and spine at 24 weeks and 2 years. High baseline osteocalcin levels and early increases in BAP were consistently associated with these increases in BMD.

Acknowledgements: The authors wish to thank the patients, investigators and study personnel who made the ASSERT trial possible. We acknowledge Joseph Marini, Eva Silvestro and Elizabeth Lee-Rykaczewski of Centocor Research and Development, Inc. for their laboratory expertise, Scott Newcomer of Centocor, Inc. for his expertise and assistance with the development of the manuscript, and Stephen Xu of Centocor, Inc. for his biostatistical support.

Funding: This study was funded by Centocor Research and Development, Inc.

Competing interests: DvdH, $A D$ and $D G B$ have received research funding and/or consulting fees from Centocor and Schering-Plough. SV, CW, DGB and JH are employees of Centocor and own Johnson \& Johnson stock.

\section{REFERENCES}

1. Marhoffer W, Stracke H, Masoud I, Scheja M, Graef V, Bolten W, et al. Evidence of impaired cartilage/bone turnover in patients with active ankylosing spondylitis. Ann Rheum Dis 1995;54:556-9.

2. Yilmaz N, Ozaslan J. Biochemical bone turnover markers in patients with ankylosing spondylitis. Clin Rheumatol 2000;19:92-8.

3. Franck $\mathbf{H}$, Keck E. Serum osteocalcin and vitamin D metabolites in patients with ankylosing spondylitis. Ann Rheum Dis 1993;52:343-6.
4. Speden DJ, Calin Al, Ring FJ, Bhalla AK. Bone mineral density, calcaneal ultrasound, and bone turnover markers in women with ankylosing spondylitis. J Rheumatol 2002;29:516-21.

5. Grisar J, Bernecker PM, Aringer M, Redlich K, Sedlak M, Wolozcszuk W, et al. Ankylosing spondylitis, psoriatic arthritis, and reactive arthritis show increased bone resorption, but differ with regard to bone formation. J Rheumatol 2002;29:1430-6.

6. MacDonald AG, Birkinshaw G, Durham B, Bucknall RC, Fraser WD. Biochemica markers of bone turnover in seronegative spondylarthropathy: relationship to disease activity. Br J Rheumatol 1997;36:50-3.

7. Toussirot E, Ricard-Blum S, Dumoulin G, Cedoz JP, Wendling D. Relationship between urinary pyridinium cross-links, disease activity and disease subsets of ankylosing spondylitis. Rheumatology 1999;38:21-7.

8. Acebes C, de la Piedra C, Traba ML, Seibel MJ, Garcia Martin C, Armas J, et al. Biochemical markers of bone remodeling and bone sialoprotein in ankylosing spondylitis. Clin Chim Acta 1999;289:99-110.

9. Golmia RP, Sousa BD, Scheinberg MA. Increased osteoprotegerin and decreased pyridinoline levels in patients with ankylosing spondylitis: comment on the article by Gratacos et al. Arthritis Rheum 2002;46:3390-1; author reply 3392.

10. Franck H, Meurer T, Hofbauer LC. Evaluation of bone mineral density, hormones, biochemical markers of bone metabolism, and osteoprotegerin serum levels in patients with ankylosing spondylitis. J Rheumatol 2004;31:2236-41.

11. van der Heijde D, Dijkmans B, Geusens P, Sieper J, DeWoody K, Williamson P, et al. Efficacy and safety of infliximab in patients with ankylosing spondylitis: results of a randomized, placebo-controlled trial (ASSERT). Arthritis Rheum 2005;52:582-91.

12. Braun J, Deodhar A, Dijkmans B, Geusens P, Sieper J, Williamson P, et al. Efficacy and safety of infliximab in patients with ankylosing spondylitis through 2 years. Arthritis Rheum 2008;59:1270-8.

13. van der Heijde D, Landewé $R$, Baraliakos $X$, Hoouben $H$, van Tubergen $A$, Williamson P, et al; Ankylosing Spondylitis Study for the Evaluation of Recombinant Infliximab Therapy Study Group. Radiographic findings following two years of infliximab therapy in patients with ankylosing spondylitis. Arthritis Rheum 2008:58:3063-70.

14. Wanders AJ, Landewe RB, Spoorenberg A, Dougados M, van der Linden S, Mielants $\mathrm{H}$, et al. What is the most appropriate radiologic scoring method for ankylosing spondylitis? A comparison of the available methods based on the Outcome Measures in Rheumatology Clinical Trials filter. Arthritis Rheum 2004;50:2622-32.

15. Creemers MC, Franssen MJ, van't Hof MA, Gribnau FW, van de Putte LB, van Riel PL. Assessment of outcome in ankylosing spondylitis: an extended radiographic scoring system. Ann Rheum Dis 2005;64:127-9.

16. Visvanathan S, Wagner CL, Marini JC, van der Heijde D, Baker D, Gathany T, et al Inflammatory biomarkers, disease activity, and spinal disease measures in patients with ankylosing spondylitis after treatment with infliximab. Ann Rheum Dis. Published Online First: 20 July 2007. doi:10.1136/ard.2007.071605.

17. Allali F, Breban M, Porcher R, Maillefert JF, Dougados M, Roux C. Increase in bone mineral density of patients with spondyloarthropathy treated with anti-tumour necrosis factor alpha. Ann Rheum Dis 2003;62:347-9.

18. Vis M, Havaardsholm EA, Haugeberg G, Uhlig T, Voskuyl AE, van de Stadt RJ, et al. Evaluation of bone mineral density, bone metabolism, osteoprotegerin and receptor activator of the NFkappaB ligand serum levels during treatment with infliximab in patients with rheumatoid arthritis. Ann Rheum Dis 2006;65:1495-9.

19. Lange U, Teichmann J, Muller-Ladner U, Strunk J. Increase in bone mineral density of patients with rheumatoid arthritis treated with anti-TNF-alpha antibody: a prospective open-label pilot study. Rheumatology 2005;44:1546-8.

20. Bernstein M, Irwin S, Greenberg GR. Maintenance infliximab treatment is associated with improved bone mineral density in Crohn's disease. Am J Gastroenterol 2005:100:2031-5.

21. Bronson WD, Walker SE, Hillman LS, Keisler D, Hoyt T, Allen SH. Bone mineral density and biochemical markers of bone metabolism in ankylosing spondylitis. $J$ Rheumatol 1998;25:929-35.

22. Mitra D, Elvins DM, Collins AJ. Biochemical markers of bone metabolism in mild ankylosing spondylitis and their relationship with bone mineral density and vertebral fractures. J Rheumatol 1999:26:2201-4.

23. Abreu MT, Geller JL, Vasiliauskas EA, Kam LY, Vora P, Martyak LA, et al. Treatment with infliximab is associated with increased markers of bone formation in patients with Crohn's disease. J Clin Gastroenterol 2006:40:55-63.

24. Torikai E, Kageyama Y, Takahashi M, Suzuki M, Ichikawa T, Nagafusa T, et al. The effect of infliximab on bone metabolism markers in patients with rheumatoid arthritis. Rheumatology 2006;45:761-4. 